\title{
Three-Stage Approach to Ultrasound Contrast Detection
}

\author{
Johan Kirkhorn, Student Member, IEEE, Peter J. A. Frinking, \\ Nico de Jong, Associate Member, IEEE, and Hans Torp, Member, IEEE
}

\begin{abstract}
A new method for detecting ultrasound contrast agents using a three-stage pulsing sequence is proposed. The method is based on observations showing that the scattering properties of contrast agents are modified by ultrasonic insonation at high power, but remain unchanged at low power. The objective of the first stage of the pulsing sequence is to use low power pulses to obtain a high resolution reference image without altering the agent. Higher power pulses in the second stage modify the contrast agent. The third stage detects the changes imposed to the contrast agent using low power pulses. A temporal filter is proposed to discriminate contrast response from clutter signal. The method is similar to power Doppler methods in that it uses several pulses to survey the target while destroying the agent. The new idea is to separate detection and destruction to circumvent a trade-off between sensitivity and resolution. Results from in vitro experiments with three different contrast agents are presented. The results are compared with harmonic power Doppler processed from the same data and show that an improvement in sensitivity is achievable by including the high power burst in the pulsing sequence. The results also show that the proposed filter reduces clutter artifacts from moving tissue.
\end{abstract}

\section{INTRODUCTION}

$\mathrm{T}$ HE PRINCIPLE of ultrasound contrast agents currently available for medical diagnostic use is to introduce small gas bubbles in the blood pool to enhance the returned signal from blood. The actual composition of the different available agents varies, but a common property is that the gas bubbles are encapsulated or otherwise stabilized to prolong their lifetime after injection. The stabilized bubbles can be altered by exposure to ultrasound pulses at insonation levels used in diagnostic ultrasound. Depending on the agent and the insonating pulse, the changes include deformation or breakage of the encapsulating or stabilizing material, generation of free gas bubbles, reshaping or resizing of gas volumes (including splitting of gas bubbles or fusion of bubbles into larger gas bubbles), or a combination of these effects [1]. The sum of the induced changes will alter the acoustic scattering properties of the contrast agent, and, by subjecting the contrast agent to multiple

Manuscript received May 31, 2000; accepted January 16, 2001.

J. Kirkhorn and H. Torp are with the Department of Physiology and Biomedical Engineering, Norwegian University of Science and Technology, Trondheim, Norway. (e-mail: hans.torp@medisin.ntnu.no).

P.J.A. Frinking and N. de Jong are with the Department of Cardiology and Experimental Echocardiography, Thoraxcenter, Erasmus University Rotterdam, Rotterdam, The Netherlands. ultrasound pulses, the changes in received echoes can be detected.

The total change induced by a single ultrasound pulse depends on several factors. Changes will increase with higher acoustic pressure amplitudes and longer pulses [2]. The pressure amplitude is bounded by safety limitations; long pulses degrade spatial imaging resolution. A frequency dependency also exists, where lowering the pulse frequency increases destruction of contrast bubbles. However, at low insonation power levels, contrast particles are able to sustain insonation without significant (i.e., detectable) changes for a time window sufficiently long to perform an imaging procedure consisting of several pulses transmitted sequentially.

Harmonic power Doppler (HPD) is currently known as one of the most sensitive techniques for detecting ultrasound contrast agents. In practice, HPD works by transmitting multiple pulses toward the object to be imaged and detecting the pulse-to-pulse changes in the received echo signals. Second harmonic bandbass filtering is applied to the received signals to exploit the nonlinear behavior of scattering from bubbles [3]. HPD operates best at high output levels because of increased contrast destruction, and pulse amplitudes close to the maximum allowed are used much of the time. However, at high output levels, nonlinear sound propagation will cause significant harmonic components from tissue [4], and the contrast agent-to-tissue ratio will decrease.

Additionally, the limited bandwidth of available transducers restricts the choice of transmit frequency to the lower end of the transducer bandwidth to ensure sufficient reception sensitivity for the second harmonic band. With these restrictions on frequency and amplitude, using longer pulses is the only way to increase destruction. However, the long pulses reduce the spatial resolution of the imaging system, and a trade-off sacrificing either resolution or sensitivity must be made.

Because HPD works by destroying contrast agent, a pause must be allowed between image frames to ensure refilling of fresh contrast agent to the object. This is known as intermittent imaging mode [5], and, in cardiac imaging, this is achieved by synchronizing the imaging to the electrocardiogram (ECG) signal. To minimize motion artifacts, imaging is gated to the phases in the cardiac cycle with least motion, usually end-systole or end-diastole.

Experiments show that, for contrast agents with a stiff shell such as Quantison ${ }^{\mathrm{TM}}$ (Quadrant Ltd., Nottingham, UK), a transient increase in backscatter is observed after 
the agent has been insonated by a high power pulse [6], [7]. This is explained as a rupture of the bubble shell by the high power pulse, followed by the release of free gas bubbles, which are more efficient scatterers than the encapsulated bubble. The increase is transient because of a decay in backscatter as the free gas dissolves into the surrounding fluid.

Based on these findings, a new detection strategy for ultrasound contrast agents has been proposed by Frinking et al. [8]. The novel approach is to first transmit a low power detection pulse to get a reference signal. The detection pulse is chosen for optimal imaging resolution and minimal contrast destruction. Then, a high power destruction pulse, called the release burst, is transmitted to destroy as much contrast as possible and to release free gas bubbles. Because the burst is not used for imaging, it does not have a limitation on length, and the frequency can be tuned with no regard for reception sensitivity in the harmonic band. Finally, a second low power detection pulse is transmitted to detect the changes introduced by the release burst. Based on temporal correlation analysis applied to the detection pulses, the method would produce a contrast-specific signal.

The release burst approach has obvious similarities to power Doppler methods in that multiple pulses are transmitted along the same line of sight, and the changes from pulse to pulse are detected. The difference lies in the separation of the destruction and detection, which circumvents the need to sacrifice either sensitivity or resolution. In this paper, the strengths of release burst and power Doppler are combined. The number of detection pulses before and after the release burst is increased, and a new clutter filter suitable for the new pulsing sequence is proposed. The clutter filter is based on polynomial regression filters [9].

This paper is organized as follows. The basic concepts of HPD are summarized first, including the inherent resolution/sensitivity trade-off of HPD. Next, the new pulsing sequence to overcome some of the limitations of HPD is described. Thereafter, a signal model for contrast and tissue based on observed contrast agent behavior is presented. Based on the model, the concepts of a filter using polynomial prediction is described. Finally, the results from an in vitro experiment with three different contrast agents with and without simulated tissue motion are presented.

\section{THEORY}

\section{A. HPD}

HPD for contrast detection exploits two different characteristics of the contrast agents, nonlinear scattering and active modification of the scattering properties of the bubbles.

From physical modeling of oscillating gas bubbles in fluids [10], contrast bubbles are predicted to emit nonlinear echoes when insonated by ultrasound. The nonlinear scattering from oscillating bubbles led to the invention of

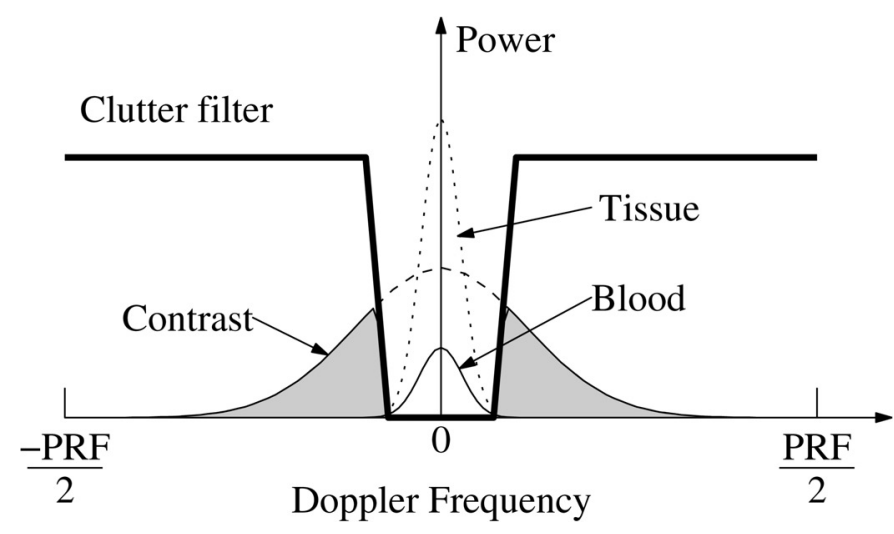

Fig. 1. Illustration of contrast in the Doppler domain. Contrast enhances the signal from blood, and destruction of the agent causes a broadening of the Doppler spectrum, which gives HPD signal even for low flow velocity.

second harmonic imaging, which has proven to enhance the signal from contrast agents significantly [3]. However, there is also a harmonic component in the echoes from tissue when no contrast is present. The harmonic component from tissue is mainly due to nonlinear propagation of the ultrasound pulse followed by linear scattering [4] or linear reflection of transmitted signals in the harmonic band. Although harmonic imaging amplifies the contrast signal in ultrasound images, the harmonic signature is not a unique contrast property.

Another property of contrast agents is that the bubbles are disrupted when insonated by ultrasound pulses at pressures used in diagnostic imaging [1], [2]. The active modification of the bubbles causes changes in echoes from pulse to pulse, and the HPD processing algorithms interpret the changes as Doppler signals. This means that the detected HPD signal from contrast agents is not a Doppler signal in the sense of motion, but a measure of decorrelation produced by both motion and changes in the contrast agent itself.

For imaging of perfusion of the heart and other organs, the blood velocities in the smallest capillary vessels are on the scale of millimeters per second. This is less than the tissue velocity of the myocardium, and the signals cannot be differentiated on the basis of velocity. The situation in the Doppler domain is illustrated in Fig. 1. In the plot, signal power is shown as a function of Doppler frequency in fractions of the pulse-repetition frequency (PRF). The signal spectrum from tissue is a strong narrow peak centered at zero frequency. When the blood velocity is low, the Doppler spectrum from blood is also centered near zero Doppler frequency, but it is much weaker than the tissue signal. Addition of contrast increases the signal strength of blood, and destruction of contrast widens the Doppler spectrum [11]. Because of the spectral broadening, some of the signal from contrast will pass the clutter filter. The degree of spectral broadening depends on the rate of destruction, meaning that efficient destruction of contrast agent is essential for the sensitivity of power Doppler methods. 


\section{B. Destruction of Contrast Agents}

Experiments show that a single ultrasound pulse's ability to destroy or modify contrast increases with amplitude and pulse length. Destruction also increases with decreasing frequency if transmit amplitude and number of cycles are kept constant. This means that the optimal pulse for destruction should be a long pulse with low frequency and high amplitude.

On modern ultrasound scanners, the amplitude of an ultrasound pulse is usually indicated by the mechanical index (MI) [12]:

$$
M I=\frac{P_{n e g}}{\sqrt{f}}
$$

where $P_{n e g}$ is the peak negative pressure in MPa and $f$ the frequency in $\mathrm{MHz}$. The MI relates to the potential to cause mechanical effects, such as cavitation and destruction of contrast bubbles. The energy in a $N$-cycle sinusoidal pulse $p(t)$ with frequency $f$ and amplitude $P$ can be found as

$$
|p(t)|^{2}=\int_{0}^{N}(P \sin (2 \pi f t))^{2} d t=\frac{N \cdot P^{2}}{f} \approx N \cdot M I^{2}
$$

Increased contrast destruction at low frequencies can be explained by increased MI and energy. Other issues not discussed here that contribute to the frequency dependency are resonance properties of the contrast bubbles and frequency-dependent attenuation. Lowering the frequency reduces the propagation attenuation in tissue and gives higher acoustic pressure at the target with the same transmit pressure amplitude. The frequency-dependent attenuation is accounted for in the estimation of MI on ultrasound scanners.

In summary, for HPD imaging, where MI and transducer bandwidth are the limiting factors for amplitude and frequency, increasing the pulse length $N$ is the only way to increase destruction to improve sensitivity. However, longer pulses will degrade the spatial resolution, and a trade-off between resolution and sensitivity must be made.

\section{Three-Stage Pulsing Sequence for Contrast Detection}

The new method described in this paper consists of a pulse sequence divided in three separate stages. The pulse sequence is illustrated in Fig. 2. The first stage consists of a number of low amplitude detection pulses transmitted to achieve a high resolution reference image of the object. The second stage is a high power release burst designed to destroy as much contrast as possible with no regard for imaging resolution. The third stage contains an additional number of detection pulses that detect the changes imposed by the second stage. The individual pulses are transmitted with a given PRF, and the total observation period is on the scale of milliseconds.

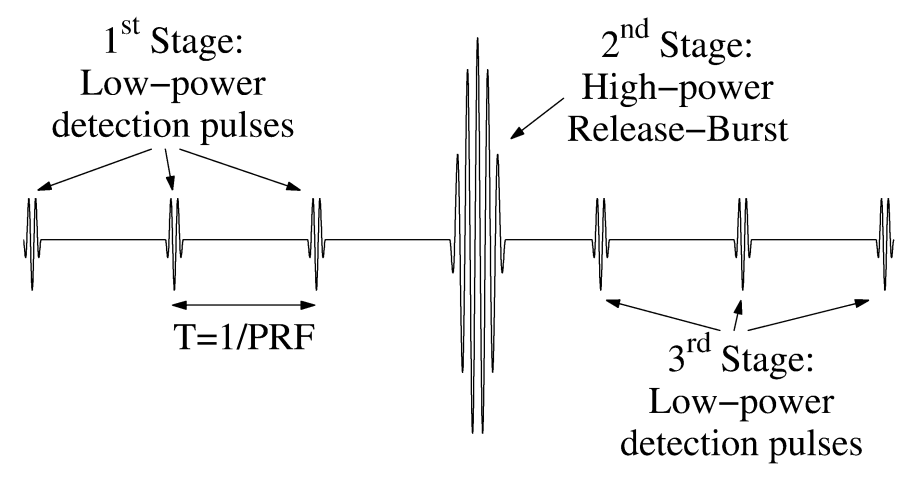

Fig. 2. Three-stage pulsing sequence. The first and third stages consist of low power pulses used to survey the target without modifying the contrast agent. The second stage is a destructive burst used to impose changes on the contrast.

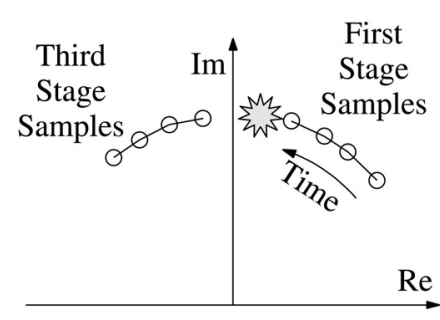

a)

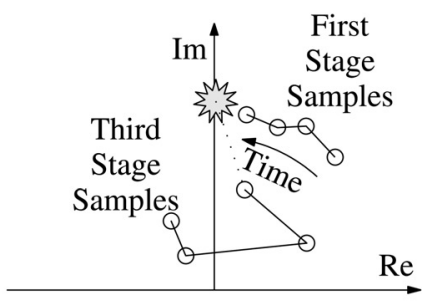

b)
Fig. 3. Signal model illustrating the expected signals from moving tissue (a) and contrast agent (b). The plots show complex demodulated samples from one depth range for successive pulses during the three-stage pulse sequence. The high power stage is illustrated by a flash.

\section{Signal Model}

Because of the fragility of contrast bubbles, tissue and contrast bubbles will behave differently during the threestage insonation sequence. It is assumed that the scattering properties of tissue are unchanged by the high power stage of the pulse sequence, and any changes in echoes from tissue in the pulses in the first and third stages are related to motion. For a rigid shell contrast agent, such as Quantison $^{\mathrm{TM}}$, the scattering properties will also be constant during the first stage, although minor agent modifications by the low power pulses cannot be excluded. The high power stage will modify the scattering properties of the bubbles, and a different response from the contrast agent is expected afterward.

Fig. 3 illustrates the signal model from moving tissue (a) and contrast agent (b). The signals are demodulated to complex baseband signals, and samples from one depth range are plotted in the complex plane. For the tissue signal (a), the samples follow the same smooth curve in the complex plane both before and after the release burst, and the phase shifts between samples originate from tissue motion. On the other hand, the samples from contrast (b) follow an almost deterministic path before the release burst but have an unpredictable transient behavior after the release burst. This is explained by the disruption of 


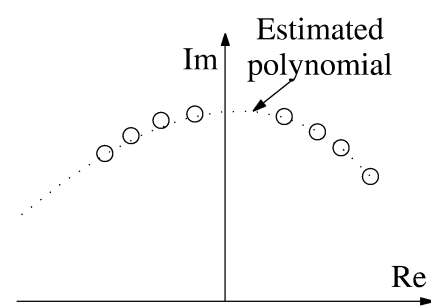

a)

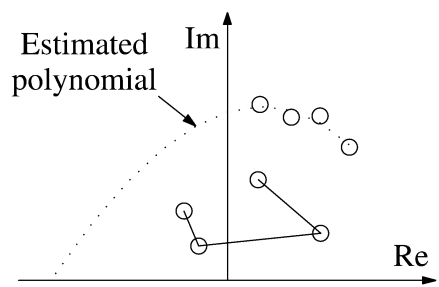

b)
Fig. 4. Principle of polynomial prediction filtering. A polynomial is fitted to the observed samples before the release burst and used to predict the samples after the burst. In this illustration, a secondorder polynomial is used. For moving tissue (a), the predicted polynomial follows the observed samples. For the contrast agent (b), the observed samples deviate from the predicted curve after the burst.

the bubble shell followed by release of the gas contained inside. The free gas bubbles are efficient scatterers, but they gradually dissolve and disappear when imaged by the low power pulses.

\section{E. Polynomial Prediction Filter}

Efficient clutter filters for color flow and power Doppler imaging can be based on polynomial regression [9]. The stationary clutter component is estimated by fitting a polynomial to the observed samples through regression. The additional release burst requires a slightly different approach. The clutter polynomial is estimated from the samples before the release burst. This polynomial curve is then extended to predict the samples after the release burst, and the power of the difference between the predicted polynomial and the observed samples after the release burst is used as a contrast-specific signal. The filtering is illustrated in Fig. 4. In the situation with moving tissue (a), the observed samples after the release burst follow the polynomial. For the signal from contrast (b), the observed samples after the release burst deviate from the estimated curve.

Both prediction and subtraction are linear operations, meaning that the proposed filtering operation can be expressed as a linear operation on the observed samples. Let $\vec{x}$ denote the vector of observed complex-demodulated samples from the same direction and depth taken at times defined in the vector $\vec{t}$. Furthermore, divide the signal and time vectors in the samples before the release burst $\left(\overrightarrow{x_{1}}\right.$ and $\left.\overrightarrow{t_{1}}\right)$ and the samples after the release burst $\left(\overrightarrow{x_{2}}\right.$ and $\left.\overrightarrow{t_{2}}\right)$. Fitting an Nth-order polynomial to the samples before the release burst, then equals finding the coefficients $a_{0}, \ldots a_{N}$, which best fit the polynomial $p(t)=\sum_{n=0}^{N} a_{n} t^{n}$ to the observed samples $\overrightarrow{x_{1}}$ taken at $\overrightarrow{t_{1}}$. The problem can be formulated as a vector equation:

$$
a_{0}\left[\overrightarrow{t_{1}}\right]^{0}+\cdots+a_{N}\left[\overrightarrow{t_{1}}\right]^{N}=\overrightarrow{x_{1}} .
$$

$\left[\overrightarrow{t_{1}}\right]^{n}$ denotes column vectors found by taking each element of the time vector $\overrightarrow{t_{1}}$ to the power of $n$. If the number of samples in $\overrightarrow{x_{1}}$ is greater than $\mathrm{N}+1,(3)$ becomes incon- sistent, and the solution must be approximated. Rewriting (3) on a matrix notation and defining the matrix $T_{1}$ with columns $\left[\overrightarrow{t_{1}}\right]^{n}, n=0 . . N$, and the coefficient vector $\vec{a}$ gives

$$
\underbrace{\left[\left[\overrightarrow{t_{1}}\right]^{0} \cdots\left[\overrightarrow{t_{1}}\right]^{N}\right]}_{T_{1}} \underbrace{\left[\begin{array}{c}
a_{0} \\
\vdots \\
a_{N}
\end{array}\right]}_{\vec{a}}=\overrightarrow{x_{1}} .
$$

To solve with respect to $\vec{a}$, the equation is multiplied by the transpose of $T_{1}$ :

$$
T_{1}^{T} T_{1} \vec{a}=T_{1}^{T} \overrightarrow{x_{1}}
$$

Because the columns of $T_{1}$ are linearly independent, the matrix $T_{1}^{T} T_{1}$ is invertible, and an expression for $\vec{a}$ is obtained:

$$
\vec{a}=\left(T_{1}^{T} T_{1}\right)^{-1} T_{1}^{T} \overrightarrow{x_{1}}
$$

This is a least squares solution to the problem. Having found the polynomial coefficients, it is straightforward to get an expression for the predicted samples $\hat{\overrightarrow{x_{2}}}$ after the release burst:

$$
\hat{\overrightarrow{x_{2}}}=a_{0}\left[\overrightarrow{t_{2}}\right]^{0}+\cdots+a_{N}\left[\overrightarrow{t_{2}}\right]^{N} .
$$

By defining the matrix $T_{2}$ similar to $T_{1}$ and inserting for $\vec{a}$, the expression is reduced to multiplication with a single matrix $B$ :

$$
\hat{\overrightarrow{x_{2}}}=\underbrace{\left[\left[\overrightarrow{t_{2}}\right]^{0} \cdots\left[\overrightarrow{t_{2}}\right]^{N}\right]}_{T_{2}} \vec{a}=\underbrace{T_{2}\left(T_{1}^{T} T_{1}\right)^{-1} T_{1}^{T}}_{B} \overrightarrow{x_{1}} .
$$

The resulting signal vector $\vec{y}$ is then found as the difference between the predicted samples $\hat{\overrightarrow{x_{2}}}$ and observed samples $\overrightarrow{x_{2}}$ after the release burst:

$$
\vec{y}=\overrightarrow{x_{2}}-\hat{\overrightarrow{x_{2}}}=\overrightarrow{x_{2}}-B \overrightarrow{x_{1}}
$$

More generally, it can be expressed as a linear filtering operation on the entire signal vector:

$$
\vec{y}=\underbrace{[-B \mid I]}_{A} \underbrace{\left[\begin{array}{l}
\overrightarrow{x_{1}} \\
\overrightarrow{x_{2}}
\end{array}\right]}_{\vec{x}}=A \vec{x} .
$$

The elements of the filter matrix $A$ are real valued, and only depend on the polynomial order $N$ and the time vectors $\overrightarrow{t_{1}}$ and $\overrightarrow{t_{2}}$. The structure of the matrix makes the method suitable for implementation on available ultrasound scanners. The power of the contrast signal $\vec{y}$ can be found as

$$
|\vec{y}|^{2}=\vec{y}^{*} \vec{y}=\vec{x}^{*} A^{T} A \vec{x} .
$$

Here, $\vec{x}^{*}$ denotes the conjugate transpose of $\vec{x}$. 


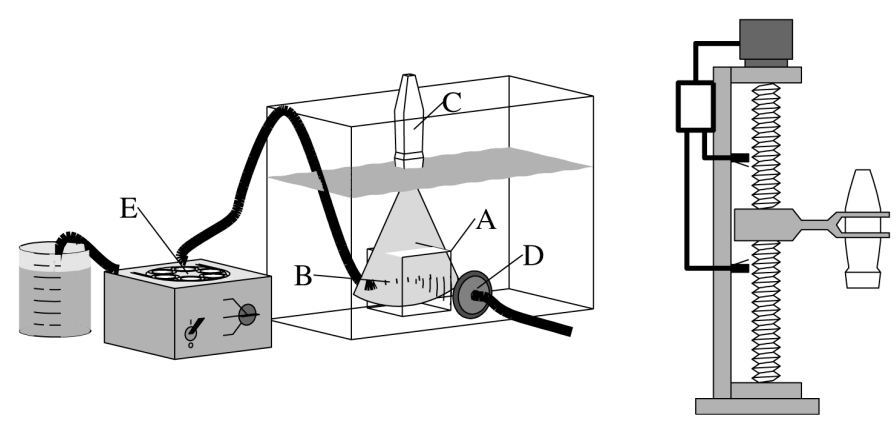

Fig. 5. Watertank setup (left) and device used to generate motion (right). Agar phantom (A), capillary fiber (B), 2.5-MHz phased array probe $(\mathrm{C}), 1-\mathrm{MHz}$ focused, single-element transducer (D), and peristaltic pump (E).

\section{EXPERIMENTS AND METHODS}

\section{A. Experimental Setup}

The method was validated in vitro in a watertank experiment (Fig. 5, left). The setup consisted of a cubic tissuemimicking phantom (A) with edges of $4 \mathrm{~cm}$. The phantom was made from agar (agar powder CMN; Boom BV, Meppel, The Netherlands) mixed with silicon carbide particles (Carborundum) of size 5 to $8 \mu m$ to get a background scattering level. The phantom material had a measured attenuation coefficient of $0.14 \mathrm{~dB} / \mathrm{cm}$ per $\mathrm{MHz}$. A hollow hemodialysis fiber (B) (Cuprophan ${ }^{\circledR}$; Akzo Nobel Fazer AG, Germany) with inner diameter $180 \mu \mathrm{m}$ was embedded in the phantom to mimic a capillary vessel. The fiber was flushed with diluted contrast agent using a peristaltic pump (E) (Varioperpex ${ }^{\circledR} 12000 ;$ LKB, Bromma, Sweden). The flow rate was $0.1 \mathrm{cc} / \mathrm{min}$, giving a flow velocity of about $4 \mathrm{~cm} / \mathrm{s}$ in the vessel.

A System Five ultrasound scanner (GE Vingmed Ultrasound, Horten, Norway) with a $2.5-\mathrm{MHz}$ phased array probe (C) (FPA $2.5 \mathrm{MHz}$ 1B; GE Vingmed Ultrasound) was used to transmit 16 low power detection pulses and receive $\mathrm{RF}$ data. The RF data were transferred digitally to a standard Pentium computer. All post-processing and visualization were done using Matlab ${ }^{\circledR}$ (The Mathworks Inc., Natick, MA).

The imaging plane of the phased array probe was carefully aligned with the capillary vessel. The distance from the probe to the vessel was $9 \mathrm{~cm}$; only the last $2 \mathrm{~cm}$ of the propagation path consisted of tissue-mimicking phantom. A focused, single-element, 1-MHz transducer (D) (Panametrics, Waltham, MA) was mounted perpendicular to the imaging plane and was used to transmit the high power release burst directed at the vessel. The trigger signal for the seventh detection pulse was captured using a digital oscilloscope (9400A; LeCroy, Chestnut Ridge, NY), which triggered an arbitrary waveform generator (LW 420A; LeCroy), producing the release burst. The waveforms were adjusted to the desired level by a 0 - to $120-\mathrm{dB}$ attenuator (355C/D; HP, Palo Alto, CA) and a 60-dB linear RF amplifier (A-500; ENI, NY). The actual pressure amplitude
TABLE I

Values of Transmission Parameters Used IN THE WATERTANK EXPERIMENTS.

\begin{tabular}{lcc}
\hline Parameter & $\begin{array}{c}\text { Detection } \\
\text { pulses }\end{array}$ & $\begin{array}{c}\text { Release } \\
\text { burst }\end{array}$ \\
\hline Frequency & $1.7 \mathrm{MHz}$ & $1 \mathrm{MHz}$ \\
Peak negative pressure & $0.5 \mathrm{MPa}$ & $1.8 \mathrm{MPa}$ \\
MI & 0.4 & 1.8 \\
Pulse length & $2 \mathrm{cycles}$ & 10 cycles \\
PRF & $4 \mathrm{kHz}$ & \\
\hline
\end{tabular}

was measured using a calibrated hydrophone (PVDFZ440400; Specialty Engineering Associates, Souqel, CA).

The imaging parameters are listed in Table I. The detection pulses were transmitted at $1.7 \mathrm{MHz}$ because this is a transmit frequency that gives good harmonic reception with the probe used. The detection pulses were chosen short, only two cycles, and at a power level verified not to destroy Quantison ${ }^{\mathrm{TM}}$ bubbles. PRF is limited by imaging depth and was chosen as high as possible. The highest MI allowed in diagnostic imaging is 1.9. The amplitude of the release burst was adjusted close to this limit, and the pulse length was set to 10 full cycles to ensure sufficient disruption of contrast. Initial studies with Quantison ${ }^{\mathrm{TM}}$ showed that the bubbles were efficiently disrupted by the selected release burst. The MI values listed in Table I are computed without derating of the measured acoustic pressures because the attenuation introduced by the phantom was considered to be negligible.

\section{B. Motion}

The previously proposed method [8] used one detection pulse before and one after the release burst. The rationale for increasing the number of detection pulses was to be able to differentiate between bubble modifications and tissue motion. To test the performance of the method in the presence of motion, experiments were performed with the phased array probe mounted in a motion device (Fig. 5, right), consisting of a DC motor connected to a screw shaft moving a probe holder vertically. Two switches on the device changed the motor rotation direction every time one of them was hit by the slide. The device produced a linear up and down motion with a speed of about $1.5 \mathrm{~cm} / \mathrm{s}$. The peak velocity of the myocardium during the cardiac cycle can reach almost $10 \mathrm{~cm} / \mathrm{s}$ [13], but intermittent imaging with contrast is usually performed during phases of the cardiac cycle where the myocardium is moving slowly. Some motion is inevitable, and the ability to suppress a motion of a few centimeters per second is necessary.

\section{Contrast Agents}

The method was tested with three different contrast agents. Suspensions of the agents were prepared as described for clinical injection and diluted in Isoton ${ }^{\circledR} \mathrm{II}$ (Coulter Electronics, Luton, UK) to get a concentration 
comparable with a clinical dose of a few $\mathrm{mL}$ of agent injected into a total blood volume of about $5 \mathrm{~L}$.

The first agent, Quantison ${ }^{\mathrm{TM}}$, consists of air-filled microbubbles with stiff and rigid shells made from human serum albumin. The stiff shell inhibits the bubbles from oscillating, and little nonlinear scattering is observed unless the pulses are destroying the shell and releasing the air [7]. Quantison ${ }^{\mathrm{TM}}$ was used in a 1:4500 dilution.

The second agent, Levovist ${ }^{\mathrm{TM}}$ (Schering AG, Berlin, Germany) is made from galactose (milk sugar) treated with palmitic acid. When Levovist ${ }^{\mathrm{TM}}$ dissolves in blood, air trapped inside the galactose is released as free gas bubbles. These bubbles have a weak encapsulating shell and are easily destroyed by ultrasound. Levovist ${ }^{\mathrm{TM}}$ was used in a 1:2500 dilution.

The third agent was BR14 (Bracco Research S.A., Geneva, Switzerland), a new experimental agent that consists of bubbles containing a high molecular weight gas enclosed by a flexible phospholipid shell. The agent shows significant nonlinear scattering and agent modification even at low insonation pressures. The dilution used was 1:4000.

Of these agents, only Levovist ${ }^{\mathrm{TM}}$ is available for clinical use. The other two are experimental agents, which are used as examples of future agents that will have more stable encapsulation than Levovist ${ }^{\mathrm{TM}}$.

\section{Processing}

For each experiment, a set of $16 \mathrm{RF}$ signals from the same direction was acquired. The RF signals were bandpass filtered for the second harmonic component and demodulated to complex baseband signals. The demodulation is a mixing of the RF signal with two sinusoid signals with $90^{\circ}$ phase difference to get the in-phase (real) and quadrature (imaginary) components of the signal. Lowpass filtering of the demodulated components allows reduction of the sampling frequency without loss of information, which reduces bandwidth for transfer and memory for storage. The complex domain is also convenient for observing phase and amplitude changes from pulse to pulse.

To compare the new method to existing HPD, each set of RF data was processed three different ways to obtain profiles of power along the beam direction (Fig. 6). First, conventional HPD processing was applied to six pulses before the release burst (a). The exact same HPD processing was applied to six pulses after the release burst (b). Finally, three pulses before and three pulses after the burst (c) were combined and processed using the polynomial prediction filtering algorithm described in this paper.

The HPD processing included a polynomial regression clutter filter [9]. For the stationary phantom experiments, polynomial order $\mathrm{N}=0$ was used. In the motion experiment, $\mathrm{N}=1$ was used. The same polynomial orders were used for the prediction filter in the new method. The clutter filter responses for six temporal samples and polynomial order $\mathrm{N}=0$ and $\mathrm{N}=1$ are shown in Fig. 7. When using $\mathrm{PRF}=4 \mathrm{kHz}$ and receiving at $3.4 \mathrm{MHz}, \mathrm{PRF} / 2$ equals a radial velocity of $45 \mathrm{~cm} / \mathrm{s}$.

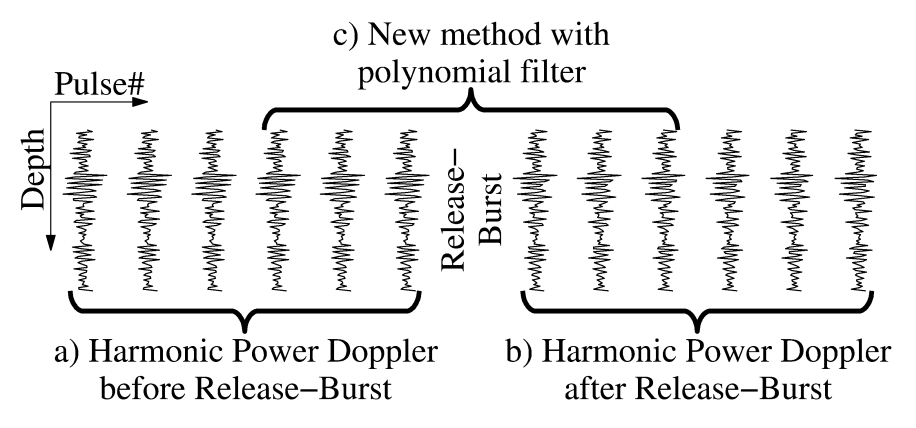

Fig. 6. Each set of RF data was processed three different ways. Six pulses before (a) and after (b) the release burst were processed separately using conventional HPD processing. Three pulses before and three pulses after the release burst were combined in the new method using a polynomial prediction filter.

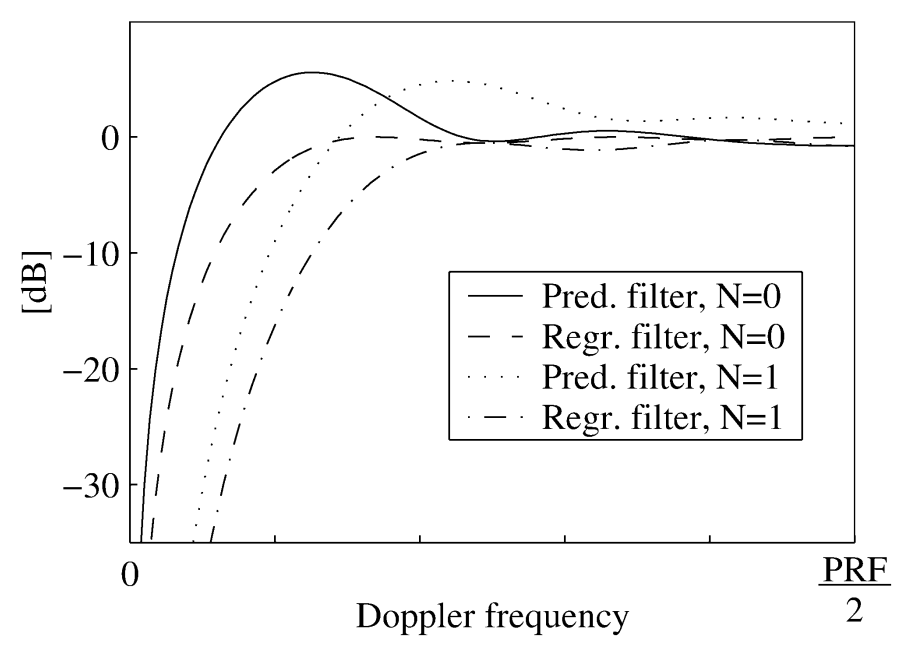

Fig. 7. Clutter filter responses. Responses of the regression filters used for HPD calculations and the prediction filters used for the new method. Six temporal samples, polynomial orders $\mathrm{N}=0$ and $\mathrm{N}=1$.

\section{E. Measure of Sensitivity}

The new method is proposed to improve resolution and/or sensitivity relative to HPD. Resolution improves because shorter pulses can be used, and sensitivity is improved by the additional signal changes introduced by the release burst. Because the same transmit pulses are used for both methods, better resolution cannot be expected in these experiments. Instead, the change in sensitivity introduced by the release burst is investigated. As a measure of sensitivity, the mean power level from the depth corresponding to the contrast-filled capillary fiber is extracted and compared with an average level in the tissuemimicking phantom. The levels and the resulting agent-totissue ratios (ATR) are indicated in the plots. Differences in the HPD profiles before and after the release burst are also expected, which can verify the assumptions about the behavior of the contrast agents and the efficacy of the release burst. A problem with in vitro experiments with contrast agents is that a stationary situation cannot easily be obtained, and results from different experiments are affected by changes in the contrast concentration caused by 
flow and destruction. Because the same data set is used for all three calculations, the results become directly comparable. The same number of pulses is also used for all calculations, although the acquisition period for the new method is longer because the release burst is transmitted between the detection pulses.

\section{Results}

The results of the watertank experiments without motion are summarized in Fig. 8. In plot A, it can be seen that Quantison ${ }^{\mathrm{TM}}$ gives no signal from the capillary with conventional HPD processing before the release burst. After the release burst (plot B), HPD gives a substantial contrast signal, about $7 \mathrm{~dB}$ above the noise level. If three pulses before and three pulses after the release burst are combined in the new method (plot $\mathrm{C}$ ), a further 18-dB increase is seen $($ ATR $=25 \mathrm{~dB})$.

Plot D shows that Levovist ${ }^{\mathrm{TM}}$ gives an ATR of about $7 \mathrm{~dB}$ with HPD before the release burst. With HPD after the release burst (plot E), the peak has almost disappeared. The new method combining three pulses before and three after (plot F) gives an ATR equal to HPD before the release burst.

Plots G, H, and I in Fig. 8 show the results obtained with BR14. Conventional HPD gives clearly visible peaks both before (plot $\mathrm{G}$ ) and after (plot $\mathrm{H}$ ) the release burst, but slightly higher after. However, when combining the samples before and after (I) in the new method, a substantial increase in ATR is gained, giving an ATR of $32 \mathrm{~dB}$.

Fig. 9 shows the results of the experiment with Quantison $^{\mathrm{TM}}$ repeated with the phased array probe mounted in the motion device moving up and down at $1.5 \mathrm{~cm} / \mathrm{s}$. Before the release burst (plot A), the fiber cannot be detected with HPD processing. After the release burst (plot B), the fiber emerges as a peak about $11 \mathrm{~dB}$ above the noise level. Both HPD curves are computed using first-order regression filters (linear regression, $\mathrm{N}=1$ ). The dashed curve in plot $\mathrm{C}$ is computed with the new method and zero-order prediction filter $(\mathrm{N}=0)$, which is merely a mean value subtraction. The ATR for the dashed curve is $5 \mathrm{~dB}$ (not indicated in the plot). When the firstorder prediction filter is applied (plot $\mathrm{C}$, solid line), the clutter is suppressed about $20 \mathrm{~dB}$, but the contrast is only attenuated $6 \mathrm{~dB}$, and the ATR increases to $18 \mathrm{~dB}$.

\section{Discussion}

For all agents used in the experiments, processing with the new method results in an ATR equal to or better than that for HPD processing on the same data.

Quantison ${ }^{\mathrm{TM}}$ was tested both with and without probe motion. The trend was the same for both experiments. Quantison $^{\mathrm{TM}}$ could not be detected with HPD before the release burst. After the release burst, the capillary could clearly be detected by HPD, which is explained by rupture of the bubble shell and release of free air bubbles, which are more easily detected than the stiff Quantison ${ }^{\mathrm{TM}}$ bubbles [6]. With the new method, the contrast-filled capillary vessel could clearly be depicted; the ATR was significantly higher than that for HPD processing.

The dashed line in Fig. 9 (plot C) illustrates the effect of motion. Motion increases the clutter level in tissue, and the peak from the capillary fiber is just above the clutter level. As a result of the low ATR, clutter peaks will create artifacts in the image. When a higher polynomial order is used in the prediction filter (solid line), the clutter is suppressed, the ATR increases to $18 \mathrm{~dB}$, and the risk of false detection artifacts is reduced.

For the currently available agent Levovist ${ }^{\mathrm{TM}}$, a definitive increase in ATR could not be achieved. The Levovist ${ }^{\mathrm{TM}}$ bubbles do not have a rigid shell and are easily destroyed, even at low insonation levels. The detection pulses before the release burst destroy some of the Levovist ${ }^{\mathrm{TM}}$ bubbles, and this explains the peak in (plot D) in Fig. 8. The release burst destroys the remaining bubbles, leaving little contrast after the burst (plot E). In an in vivo situation with more attenuation, the detection pulses will be less destructive, and the benefit of the release burst might be higher.

The results obtained with HPD before the release burst (plot G) show that the BR14 bubbles are efficient scatterers that can be modified and, thus, detected by low power insonation. The significant scattering observed from the agent with HPD after the release burst, indicates that a large portion of the bubbles survived the burst. This means that the bubbles are hard to destroy completely. Nevertheless, inclusion of an additional destruction pulse in the sequence gives a significant addition to the ATR.

It must be emphasized that the presented results are single observations selected from a limited number of experiments. Three different agents were used in the experiments, and the concentrations of the agents were not the same. This means that the absolute signal levels achieved with one specific type of processing should not be compared for different agents. Because the different processing is applied to the same sets of data, the relative performance of the methods can be judged from the results.

In the experiments, separate transducers were used to transmit the detection pulses and the release burst. For practical diagnostic imaging, the same transducer must be used to transmit all pulses. The device used to generate motion produces a linear motion parallel to the beam. In cardiac imaging, the myocardium in general does not move linearly along the beam, but exhibits an accelerated motion in all directions. However, when looking at short time intervals, the motion is approximately linear. Alternatively, more temporal samples and higher polynomial orders can be used to suppress accelerated motion. Similar to regression filters used in HPD, increasing the polynomial order $\mathrm{N}$ for the prediction filter will attenuate both contrast signal and clutter. This may reduce contrast sensitivity, but motion artifacts are also reduced, decreasing the risk of false contrast detection. The computation complexity of the proposed prediction filter is at the same level 

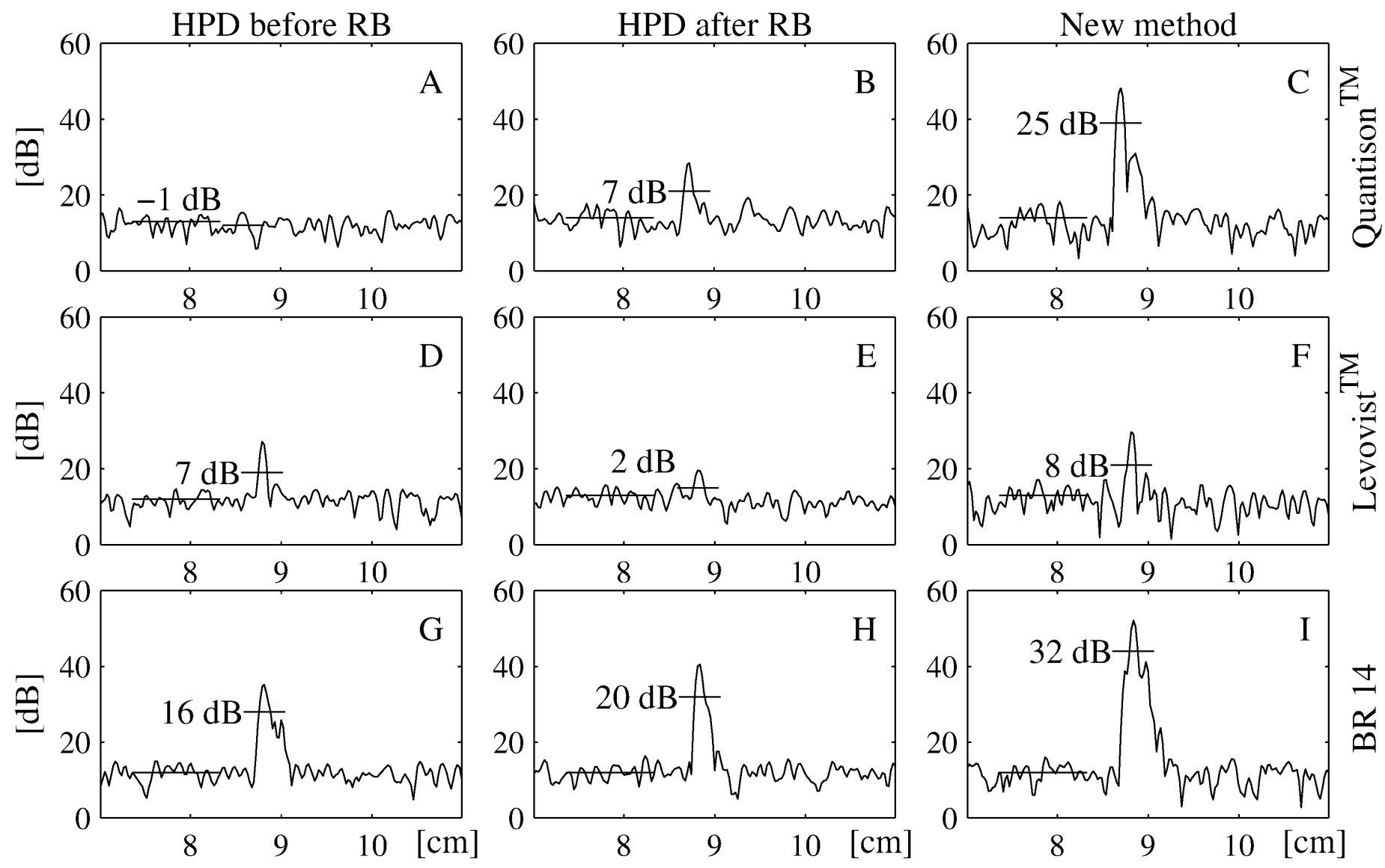

Fig. 8. Results with the imaging probe mounted in a stationary holder. Power along the beam plotted in decibels vs. depth in centimeters, with ATR indicated. The rows show results obtained with different contrast agents, viz. Quantison ${ }^{\text {TM }}$ (top), Levovist ${ }^{\text {TM }}$ (middle row), and BR14 (bottom). The columns represent different processing. The left column (A, D, and G) shows profiles obtained with conventional HPD processing applied to six samples before the release burst. The middle column (B, E, and $\mathrm{H})$ shows HPD profiles from six samples after the release burst. The right column $(\mathrm{C}, \mathrm{F}$, and I) shows profiles computed with the new method using the prediction filter applied to three samples before and three samples after the release burst.
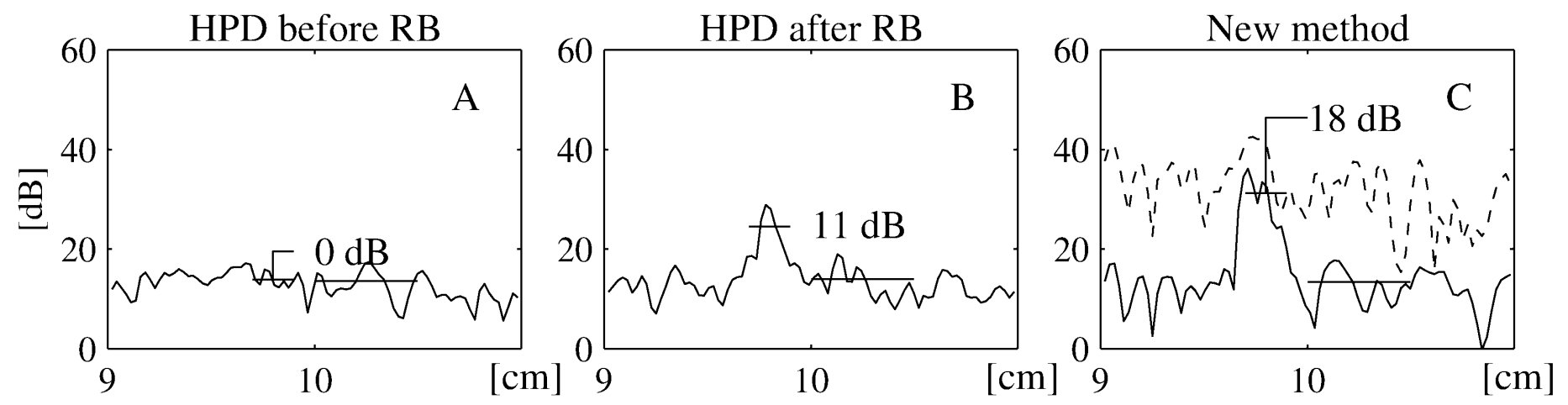

Fig. 9. Results for Quantison ${ }^{\mathrm{TM}}$ obtained with the imaging probe mounted in the motion device. The leftmost plot (A) is HPD calculated from six pulses before the release burst. The middle plot (B) is HPD calculated from six pulses after the release burst. The rightmost plot (C) shows the results of the new method using three pulses before and three pulses after the release burst. The solid line is computed by using a first-order $(\mathrm{N}=1)$ prediction filter, and the dashed line is with $\mathrm{N}=0$. 
as filters currently used for HPD, and real-time processing on modern ultrasound scanners is achievable.

HPD operates best at high power. In this respect, the low power detection pulses used in these experiments make the HPD results suboptimal. The success of HPD at higher MI is a result of more efficient bubble destruction. For Quantison $^{\mathrm{TM}}$ and BR14, the detection pulses used did not destroy the contrast bubbles. However, the same low power detection pulses destroyed Levovist ${ }^{\mathrm{TM}}$, which has weaker encapsulation than the other two agents do. Hence, the HPD results obtained for Levovist ${ }^{\mathrm{TM}}$ can be interpreted as close to optimal. The Levovist ${ }^{\mathrm{TM}}$ results show that approximately the same ATR can be obtained with HPD, as with the new method. This indicates that the potential benefit of using the new method over HPD is largest when the detection pulses do not efficiently destroy contrast bubbles. Typically, short pulses will destroy less contrast agent than long pulses. For short, low destructive pulses, the sensitivity of HPD may be improved by introducing an additional burst with more destructive characteristics. The increased sensitivity can be obtained without reducing the imaging resolution. Alternatively, shorter detection pulses and a release burst can be used to obtain the same ATR as for HPD, but with improved spatial resolution.

Finally, another benefit of using lower amplitude for the detection pulses is that the contribution to the harmonic signal from nonlinear propagation will be reduced, and the nonlinear scattering from bubbles will be more dominant.

\section{Conclusions}

Based on observations of the behavior of contrast microbubbles under ultrasonic insonation, we have proposed a new detection method for contrast agents. The method uses a three-stage pulsing sequence, which separates detection and destruction of bubbles. The sequence is matched with a polynomial prediction clutter filter, which reduces artifacts from moving tissue.

Through in vitro experiments we have evaluated the performance of the new method with three contrast agents with different characteristics. For the currently available agent Levovist ${ }^{\mathrm{TM}}$, which has a weak stabilizing shell, the performance of the new method is comparable with HPD. For future agents with more rigid encapsulation, such as Quantison ${ }^{\mathrm{TM}}$ and BR14, the new method improves the ATR in comparison with HPD computed from the same data.

In summary, the new method may give improved agentto-tissue sensitivity without increasing the power of the detection pulses or reducing spatial resolution. Alternatively, increased spatial resolution can be obtained without loss of sensitivity.

\section{ACKNOWLEDGMENTS}

Equipment and contrast agents for the experiments were provided by GE Vingmed Ultrasound AS, Bracco Research S.A., Quadrant Ltd., and Schering AG.

\section{REFERENCES}

[1] P. A. Dayton, K. E. Morgan, A. L. Klibanov, G. H. Brandenburger, and K. W. Ferrara, "Optical and acoustical observations of the effects of ultrasound on contrast agents," IEEE Trans. Ultrason., Ferroelect., Freq. Contr., vol. 46, pp. 220-232, Jan. 1999.

[2] V. Uhlendorf and C. Hoffmann, "Nonlinear acoustical response of coated microbubbles in diagnostic ultrasound," in Proc. IEEE Ultrason. Symp., vol. 2, pp. 1559-1562, 1994.

[3] P. N. Burns, "Harmonic imaging with ultrasound contrast agents," Clin. Radiol., vol. 51, Suppl. 1, pp. 50-55, 1996.

[4] M. A. Averkiou, D. N. Roundhill, and J. E. Powers, "New imaging technique based on the nonlinear properties of tissues," in Proc. IEEE Ultrason. Symp., vol. 2, pp. 1561-1566, 1997.

[5] T. R. Porter and F. Xie, "Transient myocardial contrast after initial exposure to diagnostic ultrasound pressures with minute doses of intravenously injected microbubbles. Demonstration and potential mechanisms," Circulation, vol. 92, no. 9, pp. 23912395, 1995.

[6] P.J.A. Frinking and N. de Jong, "Modeling of ultrasound contrast agents," in Proc. IEEE Ultrason. Symp., vol. 2, pp. 1601$1604,1997$.

[7] P.J.A. Frinking, N. de Jong, and E. I. Céspedes, "Scattering properties of encapsulated gas bubbles at high ultrasound pressures," J. Acoust. Soc. Amer., vol. 105, pp. 1989-1996, Mar. 1999.

[8] P.J.A. Frinking, E. I. Céspedes, and N. de Jong, "Multi-pulse ultrasound contrast imaging based on a decorrelation detection strategy," in Proc. IEEE Ultrason. Symp., vol. 2, pp. 1787-1790, 1998.

[9] H. Torp, "Clutter rejection filters in color flow imaging: A theoretical approach," IEEE Trans. Ultrason., Ferroelect., Freq. Contr., vol. 44, pp. 417-424, Mar. 1997.

[10] T. G. Leighton, The Acoustic Bubble. London: Academic Press, 1994.

[11] W. T. Shi, F. Forsberg, and H. Oung, "Spectral broadening in conventional and harmonic Doppler measurements with gaseous contrast agents," in Proc. IEEE Ultrason. Symp., vol. 2, pp. 1575-1578, 1997.

[12] J. G. Abbot, "Rationale and derivation of MI and TI - a review," Ultrasound Med. Biol., vol. 25, pp. 431-441, Mar. 1999.

[13] U. M. Wilkenshoff, A. Sovany, L. Wigström, B. Olstad, L. Lindström, J. Engvall, B. Janerot-Sjöberg, and B. Wranne, "Regional mean systolic myocardial velocity estimation by real-time color Doppler myocardial imaging: A new technique for quantifying regional systolic function," J. Amer. Soc. Echocardiogr., vol. 11, pp. 683-692, Jul. 1998.

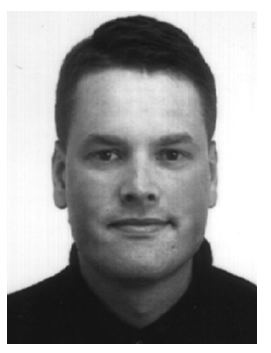

Johan Kirkhorn (S'97) was born in Eid, Norway in 1971. In 1994, he received the siv.ing. (M.Sc.) degree in electrical engineering from the Department of Telecommunications, Norwegian Institute of Technology in Trondheim, Norway. The siv.ing. thesis was written in the field of intravascular ultrasound. Since 1996, he has been a research fellow at the Department of Physiology and Biomedical Engineering, Faculty of Medicine, Norwegian University of Science and Technology in Trondheim, Norway. He is currently working toward a dr.ing. degree (Ph.D. in engineering) on a scholarship from the Norwegian Research Council and GE Vingmed Ultrasound. His research interests include signal processing and detection techniques for ultrasound contrast agents. He has been a student member of IEEE since 1997. 


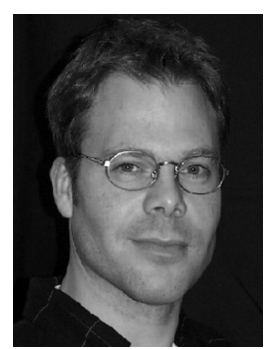

Peter J. A. Frinking was born in Bussum, The Netherlands in 1966. After secondary school he received his M.Sc. degree in applied physics from the Delft University of Technology in 1995. His M.Sc. thesis was on integration of L1 and L2 processing with application in seismic data processing. He received his Ph.D. degree in medical biophysics at the Thoraxcentre of the Erasmus University Rotterdam in 2000. Since March 2000, he has been with Bracco Research S.A. Geneva, Switzerland. His research interests include acoustic characterization of ultrasound contrast agents, ultrasound contrast imaging, and ultrasound-directed drug delivery.

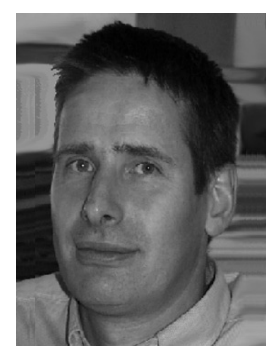

Nico de Jong (A'97) graduated in 1978 from the University of Technology in Delft, The Netherlands, where he got his M.Sc. in the field of pattern recognition. Since 1980, he has been a staff member of the Thoraxcentre of the Erasmus University, Rotterdam and University Hospital Dijkzicht. At the Department of Biomedical Engineering, he first was involved in the development of linear and phased array ultrasonic probes for medical diagnosis, especially compound and transesophageal transducers. In 1986, his interest in ultrasound applications shifted toward theoretical and practical background of ultrasound contrast agents. In 1993, he received his Ph.D for "Acoustic Properties of Ultrasound Contrast Agents." Currently, he is interested in the development of 3-D transducers and ultrasound contrast imaging. Together with Folkert Ten Cate, MD, he is organizer of the annual European Symposium on Ultrasound Contrast Imaging, held in Rotterdam, which is attended by about 175 scientists from all over the world.

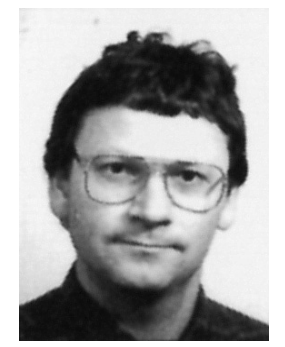

Hans G. Torp (M'93) was born in Sarpsborg, Norway in 1953. He received the M.S. and Dr.Techn. degrees from the University of Trondheim, Norway in 1978 and 1992, respectively. From 1979 to 1983 , he worked at the Division of Automatic Control, SINTEF (The Foundation for Scientific and Industrial Research of the Norwegian Institute of Technology) in Trondheim. Since 1983, he has been working at the Department of Physiology and Biomedical Engineering, Faculty of Medicine, Norwegian University of Science and Technology and since 1998 as a full-time professor. His research interests include stochastic signal/image processing with applications in ultrasonic imaging, Doppler, and color flow imaging. 